\title{
Overlap fermions on a twisted mass sea
}

\section{O. Bär}

Institute of Physics, Humboldt University Berlin, 12489 Berlin, Germany

E-mail: babrephysik.hu-berlin.de

\section{K. Jansen, S. Schaefer*, A. Shindler}

NIC, DESY, Platanenallee 6, 15738 Zeuthen, Germany

E-mail: Karl.Jansen, Andrea.Shindler, Stefan.Schaefer@desy.de

\section{Scorzato}

ECT*, Strada delle Tabarelle, 286, 38050 Villazzano (TN) Italy

E-mail: scorzatodect.it

We present first results of a mixed action project. We analyze gauge configurations generated with two flavors of dynamical twisted mass fermions. Neuberger's overlap Dirac operator is used for the valence sector. The various choices in the setup of the simulation are discussed. We employ chiral perturbation theory to describe the effects of using different actions in the sea and valence sector at non-zero lattice spacing.

XXIVth International Symposium on Lattice Field Theory

July 23-28, 2006

Tucson, Arizona, USA

\footnotetext{
* Speaker.
} 


\section{Introduction}

Dynamical overlap simulations are extremely time consuming [1]. First large scale results have been reported by JLQCD at this conference, but very large computer resources were needed to obtain them. An efficient alternative to fully dynamical simulations with chiral fermions might be provided by the so-called mixed action approach. Here, the fermion discretization employed for the sea quarks is different from the one used for the valence quarks. Provided the sea and valence quark masses are properly matched such a setup is expected to give the same continuum physics as standard unquenched lattice QCD.

The main idea is to use sea quarks which are (relatively) cheap to simulate and therefore large volumes, good statistics and small quark masses are attainable even with the fermion determinant included. Such fermion discretizations, however, typically break chiral symmetry which makes the extraction of certain observables difficult. These difficulties can be reduced by using valence quarks that fulfill the Ginsparg-Wilson equation [2] and thus respect chiral symmetry at finite lattice spacing, e.g. Neuberger's overlap fermions [3], domain wall fermions $[4,5]$ with a large extent of the fifth dimension or perfect actions [6].

One drawback of the mixed action approach is that the resulting theory is not unitary at nonzero lattice spacing. This introduces well known diseases into the theory which, however, are expected to vanish in the continuum limit. Moreover, the effects of unitarity violation can be described by mixed action chiral perturbation theory $[7,8]$.

At this conference, we present results obtained on configurations generated by the European Twisted Mass Collaboration (ETMC) [9,10]. We use an ensemble with two flavors of twisted mass fermions on a lattice of size $16^{3} \times 32$ and a lattice spacing of about $a \approx 0.1 \mathrm{fm}$. The (charged) pseudo-scalar mass is about $m_{\mathrm{PS}} \approx 300 \mathrm{MeV}$.

\section{Setup}

The lattices are generated with a tree-level Symanzik improved gauge action at $\beta=3.9$. Extensive studies have shown that this gauge action is a good compromise between ease of simulation and good short distance behavior [11].

\subsection{Twisted mass sea fermions}

We take two twisted mass sea fermions into account. The corresponding Dirac operator reads

$$
D_{\mathrm{tm}}=D_{W}^{\left(N_{f}=2\right)}\left(m_{0}\right)+i \mu \gamma_{5} \tau_{3}
$$

with $D_{W}$ the standard Wilson Dirac operator taken at un-twisted mass parameter $m_{0}$. For more details see Refs. $[12,13]$. In our simulation we set $\kappa=0.1609$ which is close to but not quite the critical value of $\kappa=0.160856$. The twisted mass parameter (which determines the bare quark mass) is set to $a \mu=0.004$.

Twisted mass QCD has many appealing properties. The fermion measure is finite and positive everywhere and, if $\kappa$ is set to its critical value, physical observables are automatically $\mathscr{O}(a)$ improved [13]. ETMC has demonstrated that twisted mass fermions can be simulated at small pseudo-scalar masses and large volume with currently available computer resources, see the talks by Jansen and Urbach at this conference [9] and Shindler at ICHEP [10]. 


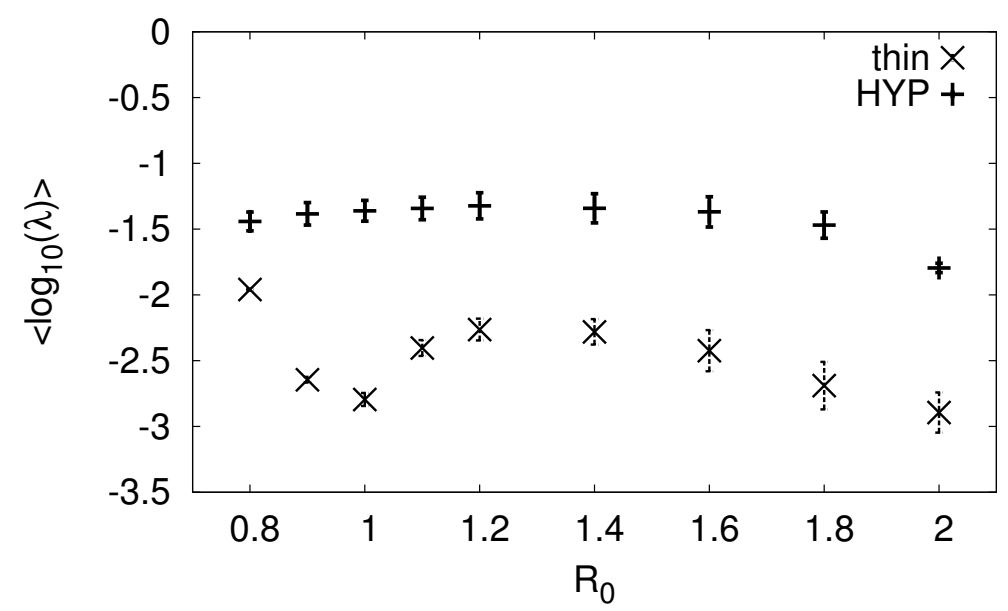

Figure 1: The effect of HYP smearing on the low-lying eigenvalues of the kernel operator $h\left(-R_{0}\right)$. We plot the average of the magnitude of 20th eigenvalue as a function of the negative mass shift $-R_{0}$. This value gives the lower bound of the rational approximation used in the construction of the overlap. The data is from an ensemble at $\beta=3.75, \kappa=0.1661$ and $a \mu=0.005$.

\subsection{Overlap valence fermions}

We use overlap fermions in the valence sector. They have exact chiral symmetry at finite lattice spacing. This ensures $\mathscr{O}(a)$ improvement at the level of the action and of the suitably chosen operators. Since also the sea quarks are $\mathscr{O}(a)$ improved, we expect small scaling violations for the whole set-up too. The overlap operator is constructed from the Hermitian Wilson Dirac operator $h=\gamma_{5} D_{W}$ which is taken at a negative mass shift $-R_{0}$.

$$
D_{\mathrm{ov}}(m)=\left(R_{0}-\frac{m}{2}\right)\left[1+\gamma_{5} \varepsilon\left(h\left(-R_{0}\right)\right)\right]+m
$$

with $\varepsilon$ the matrix sign function and $m$ the bare quark mass. The parameter $R_{0}$ is a mass at the cutoff scale which has to be tuned. The disadvantage of these fermions is the cost of the construction of the sign function. This turns out to be $\mathscr{O}(100)$ times more expensive than the application of the kernel operator.

We use the standard Hermitian Wilson Dirac operator $h\left(-R_{0}\right)$ as kernel for the overlap, which we construct from HYP blocked links [14]. Among the benefits of the HYP blocking are lower cost of construction and better locality of the overlap operator (see Fig. 1). We investigated one and two levels of stout smearing. In particular two steps can lead to a comparable situation for the overlap operator. However, the smaller range and greater (generally positive) experience with the HYP blocking lead us to use it in our production runs.

It now remains to determine the best value of the negative mass shift $-R_{0}$ of the kernel operator. We set it such that the resulting overlap operator has optimal locality. Locality means that the the norm of the operator has an exponential decay at large distances

$$
|| D_{\mathrm{ov}}(x, y ; U) \| \leq C e^{-v|x-y|}
$$




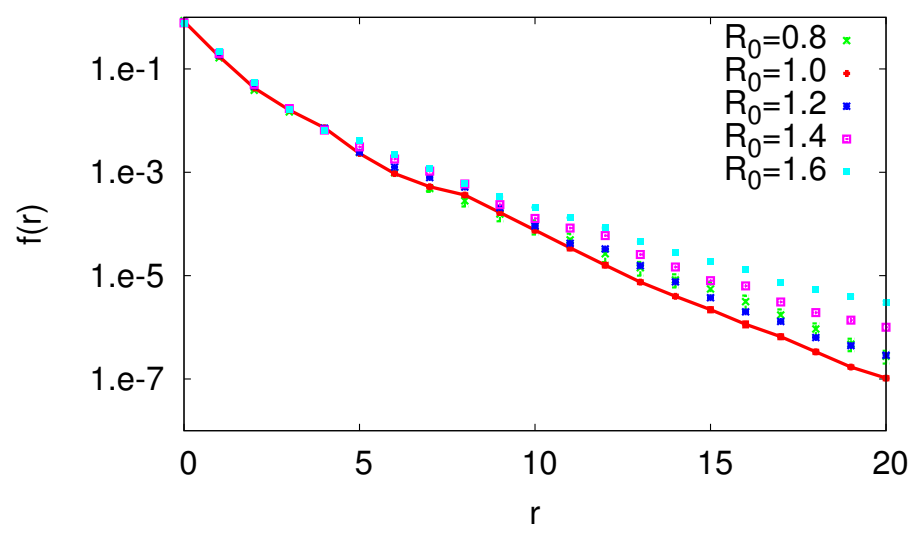

Figure 2: The locality test as defined in Eq. 2.3. We observe an exponential decay with a decay rate $v=0.607$ for the optimal choice $R_{0}=1$.

To verify this, we follow Ref. [15] and measure the exponential decay of $f(r)$ which is essentially the norm of overlap operator on a delta source.

$$
\begin{aligned}
\psi & =D_{\text {ov }} \eta \text { with } \eta(x)=\delta_{x, x_{0}} \\
f(r) & =\max \left\{\|\psi(x)\|: \operatorname{dist}\left(x, x_{0}\right)=r\right\}
\end{aligned}
$$

with dist $(x, y)$ the taxi-driver distance between $x$ and $y$. The result is shown in Fig. 2 for various choices of the parameter $R_{0}$. We find that the overlap operator has best locality properties if we set $R_{0}=1$, the free field value. (This is a well known effect of the smoothing of the gauge fields by HYP blocking.) For this choice of $R_{0}$ we measure a decay rate $v=0.607$.

\subsection{Matching}

The last parameter to fix is the valence quark mass. A simple matching condition is given by setting the masses of the pseudo-scalar mesons made of of two sea and two valence quarks equal,

$$
M_{S S}^{2}=M_{V V}^{2} .
$$

For twisted mass fermions, the neutral and the charged pseudo-scalar are not degenerate. We chose to match the charged meson mass because of its smaller statistical uncertainty. In Fig. 3 we show the valence pseudo-scalar mass as a function of the bare quark mass. This has to match the meson mass from the sea quarks whose $1 \sigma$ band is indicated by the dashed lines. The matching quark mass parameter is close to $a m_{q}=0.01$.

\section{WChPT for twisted mass LQCD}

Mixed action QCD can be described by mixed ChPT provided the pseudoscalars are sufficiently light [7]. Mixed ChPT is formulated analogously to ChPT for lattice theories with the same type of sea and valence quarks. The chiral Lagrangian through $\mathrm{O}\left(a^{2}\right)$ for untwisted Wilson sea and GW valence quarks, which is easily generalized to twisted Wilson sea quarks, can be found in Ref. [16]. 


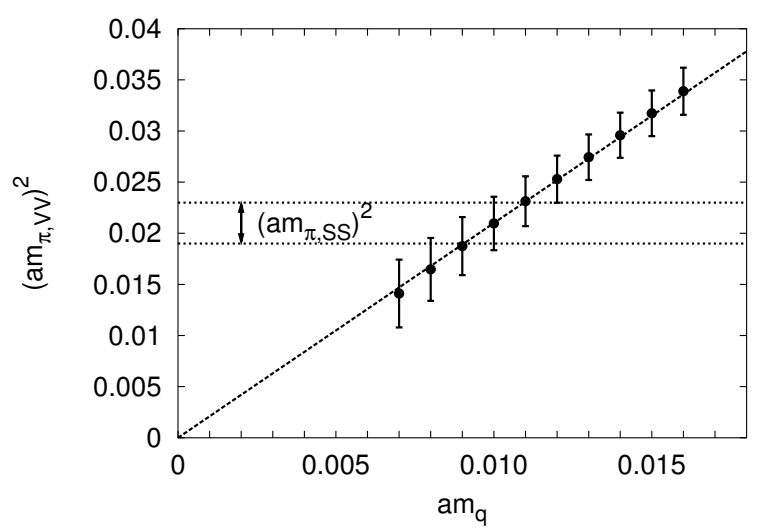

Figure 3: Matching the mass of the charged pseudo-scalar meson from the valence and the sea quarks. The dashed lines indicate the $1 \sigma$ range from the sea sector.

At leading order one finds the following expressions for the mesons masses $\left(m_{V}, \mu_{S}\right.$ denote renormalized quark masses, assuming maximal twist in the sea sector).

$$
\begin{array}{ll}
\text { Val-Val: } & M_{V V}^{2}=2 B m_{V} \\
\text { Sea-Sea: } & M_{S S}^{2}=2 B \mu_{S}, \quad \mu_{S}>\frac{2\left|c_{2}\right| a^{2}}{2 B} \\
\text { Val-Sea: } & M_{V S}^{2}=B\left(m_{V}+\mu_{S}\right)+a^{2} C_{\text {Mix }}
\end{array}
$$

The valence-valence pion mass vanishes for $m_{V}=0$ because of the exact chiral symmetry in the valence sector. The minimal sea-sea pion mass $M_{S S}^{2}=2\left|c_{2}\right| a^{2}$ is proportional to the low-energy constant $c_{2}$ [17] which determines the phase diagram for the Wilson sea quarks $[18,19]$. Note that

$$
M_{V S}^{2} \neq \frac{1}{2}\left[M_{S S}^{2}+M_{V V}^{2}\right]
$$

because of the extra low-energy constant $C_{\text {Mix }}$ present in mixed theories, and a measurement of the mixed meson mass is a direct measure for the size of the $\mathrm{O}\left(a^{2}\right)$ cut-off effects in the mixed theory.

Since mixed action theories are not unitary at non-zero lattice spacing, they are expected to show the same pathologies as partially quenched theories. A sensitive probe for these pathologies is the flavor non-singlet scalar correlator

$$
C(t)=\sum_{\vec{x}}\langle 0|\bar{d} u(\vec{x}, t) \bar{u} d(\overrightarrow{0}, 0)| 0\rangle
$$

Using the spectral decomposition the scalar correlator can be written as

$$
C(t)=A e^{-M_{a_{0}} t}+B(t)+\ldots,
$$

where $M_{a_{0}}$ denotes the mass of the $a_{0}$, the lightest $I=1$ scalar meson. $B(t)$ denotes the contribution of two pseudoscalar states (e.g. $\pi \eta^{\prime}$ ), while the ellipsis represents excited scalar states, multihadron states, etc. which are thought to be less important and therefore ignored. 


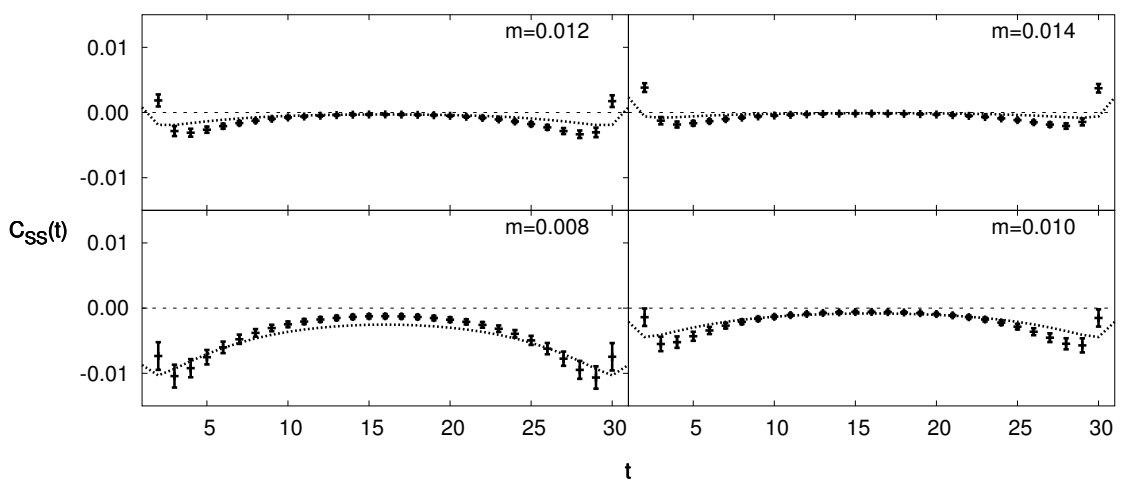

Figure 4: The scalar correlator for four values of the valence quark mass. The dotted lines represent the chiral perturbation theory prediction with the parameters determined from a fit to the data.

The contribution $B(t)$ from two pseudoscalar states has been computed in leading order (LO) ChPT including the leading cut-off effects of $\mathrm{O}\left(a^{2}\right)$ [20,21]. For large times $t$ one finds approximately $\left(N_{f}=2, M_{S S}=M_{V V}\right)^{1}$

$$
B(t) \propto \frac{e^{-2 M_{V S} t}}{M_{V S}^{2}}-\frac{e^{-2 M_{V V} t}}{M_{V V}^{2}}\left[1+\frac{\gamma_{S S} a^{2}}{M_{V V}} t\right] .
$$

The term proportional to $\gamma_{S S} a^{2} t$ dominates the large $t$ behavior. Note that, depending on the sign of the low-energy constant $\gamma_{S S}$, the scalar correlator can become negative even if the matching condition $M_{S S}=M_{V V}$ is chosen, in contrast to continuum partially quenched ChPT.

Figure $\emptyset$ shows the scalar correlator for various valence quark masses, together with a combined fit to the ChPT prediction. ${ }^{2}$ The scalar correlator is indeed negative for all valence quark masses shown. Even though ChPT is able to qualitatively describe the negative correlator, the fit is poor. One reason might be that the NLO correction are not negligible.

\section{Summary}

We presented first results from a mixed action project where we use the overlap Dirac operator in the valence sector and twisted mass fermions in the sea. We discussed the choices of the parameters of the overlap operator: the smearing of the gauge links for expedience and improved locality of the operator, the tuning of the radius of the Ginsparg-Wilson circle $R_{0}$ for optimal locality and the determination of the valence quark mass such that the pion mass in valence and sea sector match.

As physics result we discussed the scalar correlator and how to describe it with mixed action chiral perturbation theory. We found good qualitative agreement.

\footnotetext{
${ }^{1}$ For simplicity we have sent the singlet mass parameter $m_{0}$ to infinity, see Ref. [20] for its definition.

${ }^{2}$ For the fit we use the exact formula for $C(t)$ with the singlet parameter $m_{0}=1 \mathrm{GeV}$, not eq. (3.2).
} 


\section{Acknowledgments}

This work was supported in part by the DFG Sonderforschungsbereich/Transregio SFB/TR903. We gratefully acknowledge discussions with T. DeGrand, N. Garron, C. Urbach and U. Wenger. We thank T. DeGrand and the MILC collaboration [22] on whose codes parts of this work are based.

\section{References}

[1] S. Schaefer, These proceedings, BOS (LAT2006) 020.

[2] P. H. Ginsparg and K. G. Wilson, Phys. Rev. D25 (1982) 2649.

[3] H. Neuberger, Phys. Lett. B417 (1998) 141-144, [hep-lat/9707022].

[4] D. B. Kaplan, Phys. Lett. B288 (1992) 342-347, hep-lat/9206013].

[5] Y. Shamir, Nucl. Phys. B406 (1993) 90-106, hep-lat/9303005].

[6] P. Hasenfratz and F. Niedermayer, Nucl. Phys. B414 (1994) 785-814, [hep-lat/9308004].

[7] O. Bär, G. Rupak, and N. Shoresh, Phys. Rev. D67 (2003) 114505, [hep-lat/0210050].

[8] O. Bär, C. Bernard, G. Rupak, and N. Shoresh, Phys. Rev. D72 (2005) 054502, hep-lat/0503009].

[9] ETM Collaboration, K. Jansen and C. Urbach, These proceedings, POS (LAT2006) 203.

[10] ETM Collaboration, A. Shindler, ICHEP proceedings (2006).

[11] F. Farchioni et al., PoS LAT2005 (2006) 072, hep-lat/0509131.

[12] Alpha Collaboration, R. Frezzotti, P. A. Grassi, S. Sint, and P. Weisz, JHEP 08 (2001) 058, [hep-lat/0101001].

[13] R. Frezzotti and G. C. Rossi, JHEP 08 (2004) 007, hep-lat/0306014].

[14] A. Hasenfratz and F. Knechtli, Phys. Rev. D64 (2001) 034504, [hep-lat/0103029].

[15] P. Hernandez, K. Jansen, and M. Lüscher, Nucl. Phys. B552 (1999) 363-378, hep-lat/9808010.

[16] O. Bär, G. Rupak, and N. Shoresh, Phys. Rev. D70 (2004) 034508, [hep-lat/0306021].

[17] S. R. Sharpe and J. Singleton, Robert L., Phys. Rev. D58 (1998) 074501, [eep-lat/9804028].

[18] G. Münster JHEP 09 (2004) 035, hep-lat/0407006].

[19] S. R. Sharpe and J. M. S. Wu, Phys. Rev. D70 (2004) 094029, hep-lat/0407025.

[20] S. Prelovsek, C. Dawson, T. Izubuchi, K. Orginos, and A. Soni, Phys. Rev. D70 (2004) 094503, [hep-lat/0407037].

[21] M. Golterman, T. Izubuchi, and Y. Shamir, Phys. Rev. D71 (2005) 114508, [hep-lat/0504013].

[22] http://physics.utah.edu/ detar/milc.html 